\title{
Reframing Digital Storytelling as Co-creative
}

\author{
Lara Worcester
}

\begin{abstract}
This study of the digital storytelling (DST) project at the South Asia Hub of the Pathways of Women's Empowerment Research Programme Consortium examines the capacity of DST practice to articulate women's diverse experiences of empowerment, given the genre's formalities and narrative guidelines. I challenge notions that DST mediation is limited to relationships between the storyteller and the technology, and instead focus on mediation as a co-creative process. There are at least two overlooked dynamics in DST. The first is how the organisation adopts narrative guidelines to fit their framework and purpose; and second, the social relationships mediating the way actors related to one another in the workshop. I find that the workshop model and specific narrative structure may constrain ways of conveying 'experience'. Not every participant's experience or mode of narration is readily suited for DST. On the other hand, participants also report DST as useful for strengthening community relationships and opening up a more self-reflexive space for critical thinking.
\end{abstract}

\section{Introduction}

This article raises issues about the conceptual space of mediation in making audiovisual stories for research purposes by presenting findings from my case study of Pathways of Women's

Empowerment Research Programme Consortium digital storytelling (DST) project that took place at the South Asia Hub in autumn 2009. Mediation is broadly defined as the process of making and communicating meaning often in terms of technology and the storyteller's knowledge of how to use it (Peterson 2008). Digital storytelling refers to a workshop practice that produces videos of approximately two to three minutes, presenting first-person voice-over to match visual materials sourced from the storyteller's personal archives, handmade crafts, and internet stock-images, all edited together using consumer-grade computer software. Often, DST workshops are renowned worldwide for producing more 'truthful' voice because of 'minimal direct intervention by the facilitator (Burgess 2006: 207). Daniel Meadows, director of the digital storytelling BBC TV show, Capture Wales, ${ }^{2}$ writes,

No longer must we put up with professional documentaries recording us for hours and then throwing away most of what we tell them, keeping only those bits that tell our stories their own way... If we will only learn the skills of digital storytelling then we can, quite literally, 'take the power back' (2003: 192).

Meadows' statement exemplifies how DST is implicitly associated with hopes for improving democracy by eradicating 'expert' power to shape representations. However, as Nancy Thumim (2009) highlights, there is a paradox in the tendency towards technological determinism. When representations are made mediation must take place in order to make, circulate, and interpret the text. However, it is suggested (i.e. the quotation above) that mediation is minimised when people represent themselves (2009: 619).

Under-examined in DIY ('do it yourself') technology is how mediation is a collaborative activity. This study focuses on the relations of production - the roles, values, networks - that organise the appropriate and inappropriate social contexts of DST workshops. DST is not about solo work. It is a process involving multiple actors that must negotiate the rules of narrating a digital story and organisation's intentions in supporting the workshop. I investigate mediation 
at the structural level of textual genre and institutional discourse, and how actors mediate these forces together. My main research question is how does DST practice facilitate and constrain meanings attached to 'women's empowerment'?

I investigate how actors structured their stories in response to at least two commonly overlooked dynamics of the workshop process. This is not an exhaustive list. Indeed, there are other dynamics at play in mediation. But I think these help articulate why co-creativity should be acknowledged. The first dynamic is the way in which Pathways appropriates the textual features and narrative guidelines that define DST as a genre. Their appropriation is largely influenced by an institutional culture, specifically at the South Asia Hub. By 'culture', I am referring to Sally Engle Merry's definition from her ethnography work on the United Nations in which she defines culture as a 'consensual, interconnected system of beliefs and values' (2006: 6) and as a fluid 'repertoire... of ideas and practices that are not homogeneous, but continually changing because of contradictions among them or because new ideas and institutions are adopted by members' (2006: 11). Thinking about culture in this way allows one to see how discourse on issues, such as women's empowerment, is framed by an organisation. Pathways positions its purpose within the development sector with 'empowerment' as an overarching reference. I borrow the term 'shared reference' from Chandra Mohanty (2003), who argues that 'experience' (like 'empowerment') is a problematic concept in feminism as it tends to be a catch-all phrase, while afforded significant status in research. Mohanty calls for a shared frame of reference to investigate and recognise that the meanings attached to 'experience' from various historical moments are strategically important and require self-conscious inquiry of the discursive events, relationships and categories that organise how 'experience' may be understood. This is similar to the way in which Pathways approaches 'empowerment', as discussed below.

The second dynamic I examine is the social relationships that define and mediate the way actors relate to one another as media producers. The point here is that DST workshops are not isolated from relations of power that make up the greater social context. This must be considered when reflecting on the stories as ways to provide unique and textured insight into the lives of others.

\section{Context}

The DST workshop model was developed by the Center for Digital Storytelling (CDS) in California in the 1990s as part of community arts movements that treated art education as a means of mobilising people to contribute their own voices to the representation of their own and shared histories (CDS 2011: para 3). Nearly two decades following CDS's inception, the workshop is now known as a global movement with a large body of literature building on CDS director Joe Lambert's DST toolkit (Hartley and McWilliam 2009; Lundby 2008; Couldry 2008). However, hardly any research has been done on digital storytelling in the context of feminist action research. This is curious considering the large number of DST projects on gender issues, including South African non-governmental organisation (NGO), Women'sNet, which was originally trained at the CDS and subsequently trained others, including Pathways, at the 2008 Feminist Technology Exchange (Turley 2011).

Pathways' DST project is framed by a certain conception of 'empowerment'. Participants were asked to tell a story about a personal experience of struggle and how it led to positive transformation. As an overall institution, Pathways seeks to complicate neoliberal understandings of women's 'empowerment' that equate power with money and see it as something that can be achieved as a product of linear inputs and outcomes. Viewing this as a reductionist model, Pathways conceptualises empowerment as a complex process of making choices for one's self to bring about transformation and changing the conditions under which those choices are taken (Cornwall and Edwards 2010). The DST project sought to explore the influence of various factors that women face in the process of feeling empowered and the resulting dilemmas, choices, negotiations, and contexts. Both the workshop and digital stories are framed as research tools that can offer ground for theorising and depicting diverse meanings and results of 'empowerment' (internal communications, 24 March 2009).

The DST project was also an effort by Pathways to change the way development research is communicated. At least 10 per cent of its annual research programme budget was typically reserved for communications. This is indicative of the central role communication plays at Pathways in its efforts to disseminate its 
research through multiple mediums rather than depend on written academic reports alone. One of Pathways' primary purposes is to challenge stereotypes of women as well as create new representations that it considers are missing from development and mainstream media.

As part of this communication work, the Pathways team held three DST workshops. My study focuses on the first two as they were held back-to-back, lasting three days at a computer lab at BRAC University in Dhaka. My interviewees generally agreed that the first workshop was a 'training of trainers' in the hope that DST would be used in other research projects (internal communications, 19 June 2010). The participants included senior and junior researchers at both BRAC Development Institute and Pathways and Communications Officers Léa, Kristina and Akofa as storytellers; Tessa and Samia as facilitators. ${ }^{3}$

The second workshop was geared towards participants from ongoing research at the South Asia Hub. This included women of the Upazila Parishad government councils that came from rural districts just outside Dhaka, undergraduate students from different universities in Dhaka, and one woman from a taleem (women's religious lectures group). There were five facilitators in the second workshop, of which four were involved in the previous workshop, including Samia as lead facilitator and Tessa, Léa and Kristina as technical assistants.

I conducted semi-structured interviews with four Pathways Communications Officers who were DST workshop facilitators, technical assistants, and trainees-participants. As Communications Officers, these participants were important generators of the DST programme as facilitators, technical assistants, and storytellers, and offered unique insight on how DST fits in the structure of Pathways and the greater research environment. My aim was to produce dialogue, rather than a formal interview, in order to allow participants to lead in identifying what aspects of the workshop experience were challenging and beneficial. I interviewed all four participants at least once via Skype for approximately one hour and in a few cases was able to have follow-up interviews. I submitted my questions to them at least 24 hours before each interview in order to allow time to reflect on answers. I wanted to offer as much sense of comfort as possible for our discussions, considering I had never met most of them in person. This method was also particularly useful because we were discussing events which occurred over one year ago. Offering the questions in advance allowed for more time to remember, retrieve documents, and prepare more confident answers.

I conducted content analysis of Pathways' written work (in English) in order to understand the purposes, values and terms within the South Asia Hub. I read through and compared over 20 organisation reports, working papers, workshop summaries, and article publications derived from each Hub's website and various media outlets. I was also given access to some internal email correspondence between the Communications Officers regarding the DST project.

\section{Theoretical ground}

The framework of my research is largely influenced by Kelly McWilliam's 'Digital Storytelling as a "Discursively Ordered Domain", (2009) and Anna Poletti's (2011) critique of the seven narrative elements. Both scholars consider how participants structure their stories in response to the framing discourses of the DST movement. McWilliam's comparative study of two DST organisations found that narratives have different emphasis depending on how the workshop was constructed by the organisation. Her study counters popular claims in DST literature that participants produced uninfluenced or unsolicited stories independent of the context in which it was produced.

Poletti focuses on the textual instructions to produce a 'good story'. She deconstructs the seven story elements, developed by the CDS, which are the foundation of DST scriptwriting. An examination of the narrative scheme holds significant merit as DST is a genre in which firstperson narration is central to the process of creating a digital story and is given priority over other audiovisual aesthetics. Poletti claims that the seven elements 'establish expectations about the kinds of stories that will be told... and the speaking positions available to the participants' (2011: 77). Digital stories are encouraged to be told in the first person, be explicit (not ambiguous), and have resolve (the story must make a point). For example, CDS narrative element two (the dramatic question) functions as 
a way to help structure the narration and make it easy to follow. Stories which do not make their intentions clear or leave the question unresolved are not preferred. According to Poletti, this is the coaxing of a 'good story' (2011: 77-8).

Poletti's analysis is important because it is common for facilitators to incorporate the seven elements. My interviewees stated that the narrative elements were loosely referenced as workshop guidelines. However, I believe an understated aspect of DST is that while the institutional context of storymaking changes greatly and diffuses globally, the genre form of DST generally remains the same. Even as actors claim to only loosely refer to one or all of the seven narrative elements, stories typically continue to replicate emphasis on explicit meaning, resolve and closure. I examine this matter from the perspective of the Communications Officers regarding how the workshop schedule and narrative guidelines were followed and transgressed.

\section{Conveying empowerment}

Pathways' workshops were able to circumscribe a space for critical dialogue and reflection between participants. Struggle is the focal point of experience as framed by the dramatic question (narrative element two): 'What is your story of struggle that led to positive transformation?' This is exemplified by at least two digital stories that I had access to. WHAT!!! by Lopita, a Senior Researcher, was about how she achieved empowerment by defying unequal expectations between women and men in Bangladesh society. 'From Darkness to Light' by Mahmuda, a student from the taleem, overcame feelings of alienation in a culture where the niqab is uncommon. Her struggle of empowerment is one in which she draws a sense of power from her critique of society and the ability to effect change in her life by living in accordance to religious ideals.

The DST workshop resulted in stories of empowerment that yield very different life outcomes, yet both storytellers find empowerment in their subversive and oppositional position towards what they define as discriminatory. I argue that this is partly a product of the institution's own discourse on empowerment, as discussed earlier as the first dynamic of mediation. The way the South Asia Hub appropriated the dramatic question functions to bring narrative into critical discourse.
Participants were asked to answer the question: 'What is your story of struggle that led to positive transformation?' This structures the story to be reflexive and think about experience in terms of process. The digital stories represent the embodiment of political agency to respond to and reconcile one's position within social structures of power in a way that feels empowering.

Kristina (Communications Officer) found that the workshop exercises and narrative strategies facilitated her theorising. She made a story about how she dealt with the influential power she experienced as a senior rugby player in high school. Kristina viewed the seven elements and feedback exercises as important guidelines to help pinpoint where to elaborate the story in order to make certain connections more explicit. For example, Tessa (as co-facilitator) asked Kristina, 'Where is the significance of this story? You are part of a rugby team and then you become a senior player and realise how much people look up to you... so what?' This helped her examine the team dynamics and revisit some things she had done as a senior player that were advantages of power. 'It helped me form a loop of self-reflection... [it helped me] be aware of relationships around me and how we are all marked by privileges and burdens' (Hallez, personal interview, 22 June 2011). Kristina's account considers some of the narrative elements of DST to be the foundation of a reflective space that not many people are given, or able to reserve time for. This can be a useful methodology as Pathways' intent is to reconceptualise empowerment and build theory from women's standpoints.

Thus far I have examined the first dynamic of mediation by showing how the DST workshop can facilitate self-reflective and critical discourse. On the other hand, DST may constrain articulations of experience. This is exemplified by Léa's case in which she felt that the question of empowerment was deeply personal and could not be inspected through the discrete question-answer format. Léa explained that it was not due to the social dynamics of the workshop, or problems disclosing private matters to her colleagues. Rather, she felt she did not come to terms with empowerment in the way that the narrative scheme expected, which tends to discard meaning that is obscure or ambiguous. Digital stories are encouraged to be explicit and have closure, rather than open-ended statements. 
Because she found the meaning 'deeply personal' she could not bring herself to produce a story in that way. Not everyone may find it necessary to pin down empowerment as a pivotal life moment to express and be resolved via digital storytelling.

Another challenge related to the first dynamic of mediation, which occurred in the second workshop with the Upazila Parishad (UP) women. Samia had difficulty as Lead Facilitator with delegating equal amounts of sharing time to each participant during the story-circle as they tended to tell lengthy monologues compared to other participants. As politicians, these UP women were accustomed to being heard and told stories in a particular fashion that was much like campaigning. Despite time constraints, the UP women insisted on drawing out accounts. Samia's authority as facilitator was challenged and one woman ended up sharing her story for one hour.

One may interpret this as a conflict in the workshop schedule which was not prepared for, or perhaps, could not accommodate the mode through which the UP women wanted to narrate. The rules of engagement in DST emphasise that participants tell short stories focused on precise moments in one's life. The final product is restricted to three minutes long in order to 'give poignancy' and 'keep the audience's attention' (Lambert 2010: 54). At the same time, facilitators are encouraging participants to explore their creativity and choose how 'to speak for themselves' (Meadows 2003). Samia did not want to inhibit the UP women by forcing them to stop sharing. At the same time, she was concerned about other participants being compromised while mediating an intense workshop schedule.

Samia, herself, did not consider the CDS narrative scheme to be a factor of facilitation challenges. For her, the problems she faced were expressed in terms of negotiating social relations of power and difference, specifically age and occupation dynamics. This addresses the second dynamic of DST mediation: the social relations of production. Samia felt there was a hierarchy of age and professionalism which challenged her role as facilitator. As she described, these older politicians have many incredible stories of struggle as women over tremendous obstacles and there is a lot one could learn from their experiences. They entered the workshop from a certain level of authority in their communities, and that authority is attributed by a complex intersection of age, occupation, as well as gender and economic class background. One UP woman's story was about living in poverty and the courage it took to run for public office with little financial resources or emotional support from her family. Part of their profession as politicians is to understand local socioeconomic problems and campaign for solutions in a complex male-dominated bureaucratic system. Therefore, the UP women entered the workshop positioned as 'experts' in women's issues. In comparison, the facilitators and other participants were at least 15 years younger. The students were all from middle-class families in Dhaka and their digital stories generally reflected on how 'their lives were very much about being at university, struggling to study, with their whole lives in front of them' (Rahim, personal interview, 30 June 2011).

The social relations in the first workshop were also framed by power dynamics of age and occupation. However, the way in which these dynamics are configured is very different from the experience of the second workshop. All of my research participants pointed out that the process of the first workshop overturned the South Asia Hub's age and professional hierarchies.

Communications Officers were leading the project and instructing the Senior Researchers. Tessa explained, 'It was a flip of the normative hierarchy in which the younger were teaching the older' (Lewin, personal interview, 22 June 2011). Part of the disruption of the usual power dynamics was due to different relationships to digital media. The younger facilitators were more confident with the technology. All my interviewees felt that the Senior Researchers genuinely supported and invested in the new methodology. The participants did not imagine that they would ever make a video, and to learn how to do it in a short time was motivating. 'That interaction itself, in which [the Senior Researchers] were learning from the junior staff, and the fact that they were able to [commit] that amount of time out of their busy schedule is unprecedented', Tessa asserted (Lewin, personal interview, 22 June 2011). She explained the hierarchical shift had implications beyond the DST workshop that influenced the evolution of the South Asia Hub by increasing both the confidence of the younger members and the older members' respect for their work (internal communications, 14 March 2011). 
These benefits demonstrate that DST may be an excellent tool for strengthening groups with a shared agenda or shared frame of reference, such as 'empowerment', in which the actors theorise together. However, I argue that this not an innate component of the technology. Rather, it is the social context and framing discourses of its use that matter. One should be conscious of the context that gives DST meaning. The purpose of the first workshop and the shared aspirations amongst the actors had constructed DST as a mode through which researchers re-explored 'empowerment' and Pathways' way of communicating research. Hence, we may understand the first workshop as an already established collective coming together to collaborate in a new way.

\section{Conclusion}

Referring back to the research question, DST may constrain and facilitate multiple, sometime contradictory, meanings of empowerment. Not every participant's experience of empowerment or mode of narration is readily appropriate for DST. On the other hand, some participants report DST guidelines as useful tools for strengthening community relationships and opening up a more self-reflexive space for critical thinking. These are not the result of minimised mediation between the author and

\section{Notes}

1 www.pathwaysofempowerment.org (accessed 1 August 2012).

2 BBC Wales Capture Wales available at www.bbc.co.uk/wales/capturewales (accessed 12 June 2012).

3 Léa Santana is Communications Officer at the Latin America Hub in Bahia, Brazil; Kristina Hallez is Researcher and

\section{References}

Burgess, Jean (2006) 'Hearing Ordinary Voices: Cultural Studies, Vernacular Creativity and Digital Storytelling', Continuum: Journal of Media and Cultural Studies 20.2: 201-14

CDS (Center for Digital Storytelling) (2011) History [webpage], www.storycenter.org/ history.html (accessed 2 August 2011)

Cornwall, Andrea and Edwards, Jenny (2010) 'Introduction: Negotiating Empowerment', IDS Bulletin 41.2: 1-9

Couldry, Nick (2008) 'Mediatization or Mediation? Alternative Understandings of the the technology; rather, it is the social context of the workshop that facilitates a place of shared media production.

My study argues that DST mediation needs to be established more in terms of co-creativity. This is an alternative to popular terms used for 'selfmade' and 'DIY' technology. Self-representations suggest a challenge of power when institutions represent people. Some have argued, however, that it is crucial to examine the historical and political context which facilitates self-narratives, because this significantly frames how they are made, understood and read (Mohanty 2003: 77). As DST becomes more common to make and watch as sources of theorising and understanding one's self and others, analysis of the behind-thescene production is a pressing concern.

I am arguing that framing DST as a site of co-creative production can address how the narrative parameters are set up in the workshop according to intent and purpose. There needs to be recognition that the ways of telling a digital story are limited and partial accounts of 'experience' (or 'empowerment') are all standpoints. Re-evaluating the dominant textual characteristics of the genre could challenge DST into new directions.

Communications Officer at the Middle East Hub in Cairo, Egypt; Akofa Anyidoho is Programme Administrator and Communications Officer at the West Africa Hub in Legon, Ghana; Samia is Researcher and Communications Officer at the South Asia Hub in Dhaka, Bangladesh; Tessa Lewin is Communications Team Manager based at the Global Hub in Sussex, UK.

Emergent Space of Digital Storytelling', New Media Society 10.3: 373-91

Hartley, John and McWilliam, Kelly (eds) (2009) Story Circle: Digital Storytelling Around the World, Oxford: Blackwell

Lambert, Joe (2010) Digital Storytelling Cookbook, Center for Digital Storytelling, San Francisco: Digital Diner Press, www.storycenter.org/ cookbook.pdf (accessed 8 May 2010)

Lundby, Knut (2008) 'Editorial: Mediatised Stories: Mediation Perspectives on Digital Storytelling', New Media and Society 10: 363-71 
McWilliam, Kelly (2009) 'Digital Storytelling as a "Discursively Ordered Domain", in Knut Lundby (ed.), Digital Storytelling, Mediatized Stories: Self-representations in New Media, New York: Peter Lang: 147-60

Meadows, Dan (2003) 'Digital Storytelling: Research-based Practice in New Media', Visual Communications 2.2: 189-93

Merry, Sally Engle (2006) Human Rights and Gender Violence: Translating International Law into Local Justice, University of Chicago Press

Mohanty, Chandra Talapade (2003) Feminism Without Borders: Decolonizing Theory, Practicing Solidarity, Durham NC: Duke University Press

Peterson, Mark Allen (2008) Anthropology and Mass Communication: Media and Myth in the New Millennium, New York: Berghahn Books

Poletti, Anna (2011) 'Coaxing an Intimate Public: Life Narrative in Digital Storytelling',
Continuum: Journal of Media and Cultural Studies 25.1: 73-83

Thumim, Nancy (2009) “"Everyone has a Story to Tell": Mediation and Self-representations in Two UK Institutions', International Journal of Cultural Studies 12.6: 617-38

Turley, Anna (2011) The FTX (Feminist Tech Exchange) at the Forum, highlights of Association for Women's Rights in Development (AWID)'s 11 th International Forum on Women's Rights and Development, www.awid.org/Homepage/ Forum/new-forum/Feminist-Tech-Exchange/ This-is-a-report-of-the-activities-andexperiences-as-they-carried-on-to-the-AWIDForum (accessed 12 October 2011) 